\title{
Turning Microplastics into Nanoplastics: Digestive Fragmentation by Antarctic krill
}

\author{
Amanda L. Dawson*1; So Kawaguchi², Catherine K. King ${ }^{2}$, Kathy
} A. Townsend ${ }^{3}$, Rob King ${ }^{2}$, Wilhelmina M. Huston ${ }^{4}$, Susan M. Bengtson Nash ${ }^{1}$

1. Environmental Futures Research Institute, Griffith School of Environment, Griffith University, 170 Kessels Road, Nathan, QLD 4111, Australia

2. Australian Antarctic Division, Department of the Environment and Energy, 203 Channel Highway, Kingston, TAS 7050, Australia

3. School of Biomedical Sciences, Moreton Bay Research Station, University of Queensland, North Stradbroke Island, QLD 4183, Australia

4. School of Life Sciences, University of Technology Sydney, Faculty of Science, 15 Broadway Ultimo, NSW 2007 
26 Abstract

27 Microplastics (plastics $<5 \mathrm{~mm}$ diameter) are at the forefront of current environmental pollution research.

28 However, little is known about the degradation of microplastics through ingestion. Here, by exposing

29 Antarctic krill (Euphausia superba) to microplastics under acute static renewal conditions, we show the

30 first observations of physical size alteration to microplastics ingested by a planktonic crustacean.

31 Ingested microplastics $(31.5 \mu \mathrm{m})$ were fragmented into pieces $(<1 \mu \mathrm{m}$ diameter). Previous feeding

32 studies have shown spherical microplastics either; pass unaffected through an organism and are

33 excreted, or are sufficiently small for translocation to occur. We identify a new pathway; microplastics

34 were fragmented into sizes small enough to cross biological barriers, or were egested as a mixture of

35 triturated particles. These findings suggest that current laboratory-based feeding studies may be

36 oversimplifying interactions between zooplankton and microplastics but also introduces a new critical

37 role of this, and potentially other species, in the global biogeochemical cycling and fate of plastic. 
Microplastics (plastics $<5 \mathrm{~mm}$ ) have been isolated from biota representing the full spectrum of feeding mechanisms, habitats, and trophic levels from zooplankton to megafauna ${ }^{1}$. Marine microplastics are attributed to two main sources; the direct release of micro sized plastic particles into the environment and the in situ environmental breakdown of larger plastics. Microplastics are prevalent in the marine environment and degradation occurs continuously on unknown timescales until the polymer is completely mineralized into carbon dioxide, water and biomass ${ }^{2}$. All microplastics are expected to continue fragmenting, thus reaching nano sizes $(<1 \mu \mathrm{m})$. Thus microplastics in the environment are heterogeneous in size and in shape ${ }^{3}$, and consequently present a challenge for standardized monitoring ${ }^{1}$. Planktonic suspension and filter feeders may be the most susceptible to microplastic ingestion due to the relatively indiscriminate nature of this feeding strategy ${ }^{4}$. In particular, polyethylene (PE), polypropylene (PP), and expanded polystyrene (PS) are all less dense than seawater, making them buoyant and available to planktonic species ${ }^{5}$. Detrimental health effect have been associated with physical obstruction of the digestive system and associated reduced nutritional condition ${ }^{6}$.

Laboratory-based feeding studies are a commonly used approach for the quantification of exposure and associated effects. Often these studies use invertebrate species such as zooplankton, which form the basis of the pelagic food web. Ingestion at this level therefore carries a threat of possible plastic bioaccumulation and biomagnification to higher trophic levels ${ }^{1}$. Organisms are exposed to relatively homogenous, commercially available, plastic beads to replicate environmental condition ${ }^{3}$. Such studies have confirmed numerous planktonic species are capable of ingesting and egesting microplastics ${ }^{7-11}$, many of which were associated with toxic and physiological effects ${ }^{2,12-15}$. Despite a growing body of exposure and affect assessments, the ecological consequences of microplastic ingestion by zooplankton remain unclear. Further, the fate and degradation of microplastics, as a consequence of ingestion is rarely considered.

Here we expose Antarctic krill (Euphausia superba, hereafter 'krill'), a keystone species in the

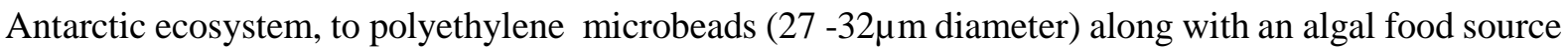
to determine the fate of microplastics ingested by a planktonic crustacean of high dietary flexibility and ecological importance. Krill predominantly feed on silica diatoms but regularly prey on other zooplankters including salps, copepods and other krill ${ }^{16}$. In terms of biomass, Antarctic krill are extremely abundant, supporting a large number of Southern Ocean predators ${ }^{17,18}$ and are a predominant phytoplankton grazer in the Southern Ocean ${ }^{18,19}$. Krill filter feed by forming a feeding basket through which water is passed (Fig. S1A). Food particles are retained on the basket and then transported to the mandibles for mastication ${ }^{20,21}$. The mandible, situated at the base of the oesophagus, is equipped with a cutting and grinding surface ${ }^{22}$. Food is then directed through the short oesophagus into the stomach and gastric mill where it is mixed with digestive enzymes for further mastication ${ }^{23,24}$. Thereafter, particles 
directed to the mid and hind gut for egestion ${ }^{25}$. Egested particles are encased in a peritrophic membrane which protects the mid and hind gut from abrasion ${ }^{26}$.

The digestive gland is the primary site for cellular digestion (Fig S1C)). The gland is made up of groups of blind ending tubules, which are comprised of epithelial cells. Food particles that enter the digestive gland are pumped into the tubules, where digestive enzymes are directly released, thus allowing for nutrient adsorption and intracellular digestion to take place ${ }^{26-28}$.

Exposed krill and their faecal material were examined microscopically to (1) quantify the size of particles present in the krill digestive system and in egested material, (2) identify where these particles are localised within the digestive system, and (3) examine the effect of particle size on egestion. We find that Antarctic krill are capable of fragmenting pristine PE microbeads into significantly smaller fragments. This is the first report of nanoplastics generated by the ingestion of microplastics in a marine species.

\section{Results}

\section{Antarctic krill fragment ingested virgin polyethylene}

To determine the effect of ingestion on microplastic beads we exposed krill to a 4 day static renewal assay, which incorporated daily feeding on two (low - 20\% and high - 80\%) PE microplastic and algal diets. Krill were exposed daily for 4 hours to their diet; this was followed by 20 hours in clean seawater. Whole krill were enzyme digested after exposure to isolate the ingested microplastics, as was faecal material collected throughout the experiment. We compared the size distribution of particles from the stock suspension to the distribution of particles within the krill and egested faecal pellets. We found all krill contained a mixture of whole PE microplastic beads and PE fragments that was not consistent with the exposure stock. Beads in the stock suspension had a mean diameter of $31.5 \mu \mathrm{m}( \pm 7.6$ Standard Deviation, S.D), whereas the mean particle size isolated from within the krill was, on average, 78\% smaller than the original beads $(7.1 \mu \mathrm{m} \pm 6.2 \mathrm{~S}$.D), with some fragments reduced by $94 \%$ of their original diameter. Particles isolated from faecal material were also reduced $(6.0 \mu \mathrm{m} \pm 5.0$ S.D). Further, the size distribution of particles within the krill, and excreted particles, were significantly different to beads in the exposure stock ( $D=112, p<0.001$ and $D=113, p<0.001$ respectively) (Fig. 1A, Fig. S3A). The reduced plastic particle size found in krill and their faecal pellets revealed that Antarctic krill were physically fragmenting beads after ingestion. We found no relationship between krill size and their ability to fragment plastics $\left(\mathrm{F}_{3,15}=2.595, \mathrm{p}>0.05, \mathrm{R}^{2}=0.357\right)$.

To ensure that the homogenization process was not responsible for fragmenting the beads we carried out procedural blanks. These consisted of whole krill enzyme digested, beads enzyme digested and beads not subjected to any digestion or homogenization. Beads were unaffected by the sample analysis procedures, neither the homogenisation process nor the digestion enzymes were responsible for fragmenting the beads. 
109 Particles from the krill and bead blanks were found to be unaffected by the enzyme digestion protocol.

110 Visually, beads from the stock suspension appeared similar to the bead blanks. As did the whole beads

111 and fragments isolated from the krill and krill blanks. The distributions of particle sizes from

112 experimental and blank samples were very similar, despite unequal sample sizes (Fig. S3). Overall it

113 was determined that krill were responsible for fragmenting the beads.

114 Repeated exposure decreases fragmentation

115 Notably, not all ingested beads were fragmented in the current study. To further explore this observation

116 we compared the proportion of fragments to whole beads isolated from whole krill homogenates and

117 faecal pellets exposed to the high and low treatments. The proportion of fragmented beads egested by

118 the krill on days 1 and 4 were compared to assess the effect of repeated exposure. An extra sample point

119 was added on day 4 to assess fine scale temporal variation within a daily cycle after repeated exposure.

120 Whole beads were found in the stomach and midgut content, as well as faecal pellets. Exposure 121 concentration played an important role in the ability of krill to fragment the PE beads; where lower plastic concentration appeared to facilitate the krill's capacity to triturate plastic. Krill contained significantly more whole beads when exposed to a high plastic diet than a low plastic diet $\left(\mathrm{X}^{2}{ }_{1}=323\right.$, $(N=67476), p<0.001)$ (Fig. 1B). Faecal pellets also followed this trend $\left(X^{2}{ }_{1}=600,(N=54670), p\right.$ $<0.001)$. Further examination revealed a significant interaction between time, dose, and the proportion of fragmented plastic $\left(\mathrm{F}_{(1,45778)}=328, \mathrm{p}<0.001\right)$. Increased dose and repeated exposure appeared to inhibit the ability of krill to triturate plastic. Faecal pellets of high dose krill collected after the first day of exposure, contained a lower proportion of whole beads than faecal pellets collected after the final day of exposure $\left(\mathrm{X}^{2}{ }_{1}=384,(\mathrm{~N}=27317), \mathrm{p}<0.001\right)$ (Fig. 3A). Whereas, when comparing the first and last day of exposure, krill exposed to low dose plastic appear capable of fragmenting plastics irrespective of repeated expose $\left(\mathrm{X}^{2}{ }_{1}=2,(\mathrm{~N}=18465), \mathrm{p}>0.05\right)$ (Fig. $\left.3 \mathrm{~A}\right)$. Faecal pellets of high dose krill collected at 4 and 24 hours on the last day of exposure clearly show an increasing trend of whole beads being egested over the final 24 hours $\left(\mathrm{X}^{2}{ }_{1}=238,(\mathrm{~N}=24828), \mathrm{p}<0.001\right)$ (Fig. 3B). The low and high dose krill both exhibit similar proportions of egested whole beads at 4 hours on the last day of exposure. However, where the high dose krill appear to decrease their ability to fragment plastics over time, the low dose krill exhibited the opposite trend over the final 24 hours. Krill exposed to the low dose egested a higher proportion of fragments suggesting more efficient fragmentation $\left(\mathrm{X}^{2}{ }_{1}=5,(\mathrm{~N}\right.$ $=17175), \mathrm{p}=0.018$ ). Overall it appeared that krill at the beginning of each daily pulse exposure were efficient at fragmentation, as krill ingested more beads the fragmentation decreases.

\section{Tissue localisation of fragments}

141 To further investigate plastic fragment kinetics within the organism, histological cryosections of 142 exposed krill were prepared. We observed microplastics within the esophagus, stomach, digestive gland and midgut of deceased krill (Fig. 2, Fig. S1). Plastic were also visible in the stomach of live krill. 
Mandibles frequently had plastic fragments enmeshed in the grinding surface. The bulk of plastic maceration presumably took place in the stomach and gastric mill, which is responsible for mechanically fragmenting food particles under normal feeding conditions. Due to their predominantly herbivorous diet, Antarctic krill have complex digestive enzymes with high activity ${ }^{18}$. In this study we did not examine the effects of digestive enzymes on microplastics thus cannot rule out the possibly that digestive enzymes did not contribute to the fragmentation displayed in this study. Small food items then pass through a filter then pass through a filter (approximately $0.14 \mu \mathrm{m}$ ) into the digestive gland. Thus, large plastic fragments and full sized beads were excluded from the digestive gland and directed to the midgut for excretion.

Microscopic limitations precluded a comprehensive investigation into the size and abundance of fragments found in the digestive gland. However, we detected particles in the digestive gland of two out of the five krill examined, within an approximate size range of $150-500 \mathrm{~nm}$. The digestive gland is responsible for the absorption of digested material into the haemolymph ${ }^{29}$. The presence of PE fragments in the digestive gland revealed krill triturate PE beads to colloidal sizes, which increases the capacity for crossing biological barriers ${ }^{30}$.

\section{Size dependent egestion}

To examine egestion, we exposed krill to low dose plastic for 10 days, after which their diet was swapped to $100 \%$ algae. Faecal pellets were collected for 5 days following the diet change. Small triturated fragments were more persistent and retained within the krill's body for longer than large beads. The proportion of whole beads excreted by krill decreased significantly throughout the egestion period $\left(\mathrm{X}^{2}{ }_{4}=16,(\mathrm{~N}=21525), \mathrm{p}=0.003\right)$, with whole beads no longer excreted after three days following the diet change (Fig.4). Fragments were present in faecal material throughout all samples. This finding corresponds well with previous observations of size dependent egestion in marine invertebrates, both in laboratory and wild caught species ${ }^{31-33}$.

\section{Discussion}

Despite a growing body of research, there are still considerable knowledge gaps regarding spatial patterns and abundance of microplastics in the marine environment. The paucity of studies concerning microplastic ingestion in wild caught zooplankton hampers comparisons to this study. Microplastics isolated from euphausiids and other zooplankton have been found to range in size from $123 \mu \mathrm{m}$ to $\leq 2000 \mu \mathrm{m}^{7,34}$, which is more than two orders of magnitude larger than the bead fragments Antarctic krill were found capable of producing in this study.

The phenomena of digestive fragmentation has never before been observed in other planktonic crustaceans, such as copepods or isopods, despite the fact that many of which possess similarly developed gastric mills and mouthparts designed for mechanical disruption ${ }^{29}$. However, copepods are theorized to scrape biofilms from the surface of pelagic plastics, inadvertently consuming liberated 
plastic fragments ${ }^{35}$. We hypothesise the absence of this observation in other planktonic laboratory crustaceans may be due to the use of different polymers in experiments. Two of the most commonly used laboratory plastics for feeding studies, PE and PS, differ in mechanical properties. The more commonly used PS is a rigid plastic, with a higher capacity to withstand stress than $\mathrm{PE}^{36}$.

Regardless of their original polymer properties, marine microplastics are largely comprised of secondary plastics, derived from the breakdown of larger plastic items ${ }^{3,37}$. These secondary plastics are subject to weathering and chemical degradation rendering them physically and chemically altered from virgin plastics, such as those used in this study. Weathering serves to reduce the mechanical strength of plastics which leaves them brittle ${ }^{38,39}$. Whilst the capacity of zooplankton to fragment secondary plastics requires further study, we suggest that embrittlement of secondary plastics will facilitate digestive fragmentation. We hypothesize fragmentation of microplastics after ingestion may be more common in the environment than the published literatures currently demonstrate. Previous observations of crabs altering laboratory degraded fibres after ingestion offer weight to this hypothesis ${ }^{40}$.

Nonetheless without further testing on other polymers and microplastic particles with varying degrees of degradation, it is difficult to speculate the frequency at which microplastic fragmentation in the environment could be occurring. Polyethylene is one of the most common plastic in the marine environment ${ }^{41,42}$, thus even if this phenomenon is restricted wholly to PE; it still could present a significant pathway of microplastic degradation in the marine environment.

In general, polyethylene has a low resistance to UV degradation and recent studies have identified that PE microplastics collected from the North Atlantic subtropical gyre were considerably weathered, with shorter polymer chain lengths, reduced molar mass and were more crystalline than reference $\mathrm{PE}^{39}$. Glassy polymers such as PS or Polyethylene terephthalate (PET), however, are stronger and less susceptible to UV degradation ${ }^{38}$. Despite the properties of pristine polymers, all plastics, even those with chemical stabilisers, will eventually degrade in the environment.

The low exposure concentration applied in this study was within the same order of magnitude as microplastic concentrations observed in pelagic systems of the North Pacific Subtropical Gyre ${ }^{2}$, which are among the highest concentrations reported globally. Limited pelagic microplastics surveys from the Southern Ocean isolated between 0.0032 and 1.18 particles $\mathrm{m}^{-343,44}$, these levels are considerably less than those used in this experiment. In spite of the elevated exposures used in these experiments, considerable bead fragmentation was achieved. These preliminary findings, although limited by scarce environmental data related to plastic $<330 \mu \mathrm{m}$ in natural marine systems, suggest that current concentrations may be within the bounds of optimal trituration for krill, but fragmentation efficiency may be affected by chronic exposure. The increased fragmentation of plastic noted at low exposure conditions gives further weight to our hypothesis that digestive fragmentation is more common in the environment than recorded in current literature, which often use similarly high exposure concentrations 
214 for exposure experiments ${ }^{3}$. Current contamination levels in the Southern Ocean are theoretically low 215 enough to promote efficient digestive fragmentation by krill species, and in a global context, possibly 216 for other zooplankton with sufficiently developed gastric mills.

217 We did not examine these fragmented particles for induced toxicological effects. Several laboratory 218 studies have demonstrated the ability of micro and nanoplastics to translocate to the haemolymph ${ }^{45-47}$, 219 however in these studies, the exposure particle size was sufficiently small to achieve translocation. We 220 identify the potential for translocation to occur after an organism has physically altered the ingested 221 plastics. This reveals a previously unidentified dynamic in the plastic pollution threat, with the 222 implication that biological fragmentation of microplastics to nanoplastics is likely widespread within 223 most ecosystems. As such, the harmful effects of plastic pollution must take into consideration not only 224 the physical effects to the individual of macro and microplastic ingestion, but also the potential cellular 225 effects of nanoplastics and the ecosystem impacts of biomagnification hereof. The effect of nanoplastics 226 on crustaceans is unknown, although previous studies observed PE microbeads to induce genotoxicity and immunological effects in haemocytes ${ }^{47}$. Previous studies have suggested relatively simplistic interactions between microplastics and zooplankton ${ }^{7,9,10}$ and biota-facilitated plastic degradation (considered to be predominantly undertaken by microorganisms) is currently considered negligible in the marine environment ${ }^{2}$. However, our results bring into question these previous conclusions. The fate of these altered particles, after egestion, death or predation is completely unknown, and is not necessarily comparable to non-ingested particles. Studies that neglect these interactions may be neglecting a significant pathway of degradation. Interestingly, ter Halle, et al. ${ }^{48}$ recently showed that smaller microplastics are fragmented faster than larger particles under environmental conditions. The repercussions of organisms accelerating this process deserve further study.

237 It is also possible that fragmentation resulted from, or was enhanced by, the presence of silica diatoms 238 in the diet. The churning and grinding action of the gastric mill combined with the sharp edges of 239 triturated algae may have fragmented the beads. This could explain the decreased fragmentation in the 240 high exposure treatments, where there was a correspondingly lower algal concentration. However, this 241 mechanism does not explain the temporal variation in fragmentation efficacy with repeated exposure, 242 as krill diet within treatments remained constant over time. Thus fragmentation may have been enhanced by the presence of silica diatoms but it was unlikely to be the sole cause of fragmentation. This study uncovered the ability of an Antarctic keystone species to physically change ingested microplastics in a manner not previously described and in doing so, provides evidence for biologically facilitated production of nanoplastics. We hypothesise fragmented microplastics have increased potential for interaction at the molecular level, as seen in other nanoplastic studies ${ }^{12}$, and this warrants significant attention to nanoparticle toxicology in the discussions surrounding global plastic pollution. 
249 Triturated microplastics will likely impact potential particle bioavailability and biomagnification, and 250 likely influence the timescales needed for complete mineralisation.

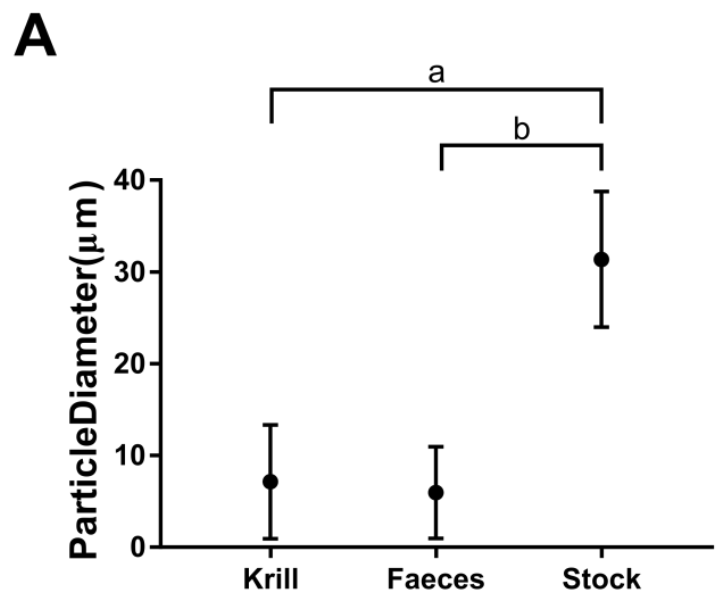

\section{B}

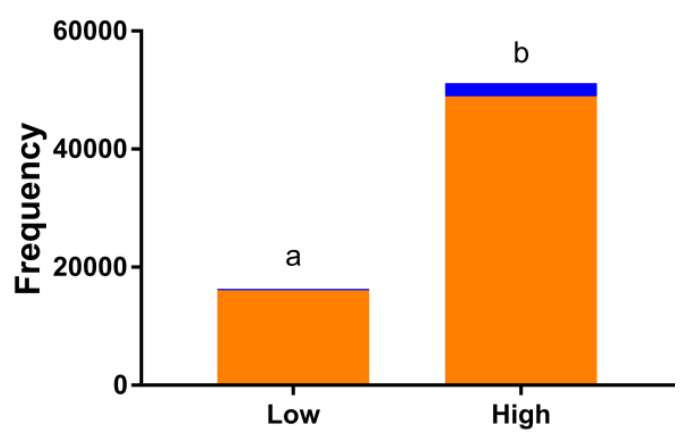

Plastic Concentration

252 Fig. 1. Size of polyethylene particles A) Microplastic particle size (mean \pm S.D) in all sample 253 types: whole krill homogenates, egested faecal pellets, and in the exposure stock suspension, 254 B) Frequency of whole beads (Blue) and fragments (Orange) isolated from Antarctic krill 
(Euphausia superba) exposed to a Low $(20 \%)(\mathrm{n}=9)$ and High $(80 \%)(\mathrm{n}=9)$ plastic

256 concentrations. Letters denote statistically significant differences $(\mathrm{p}<0.05)$
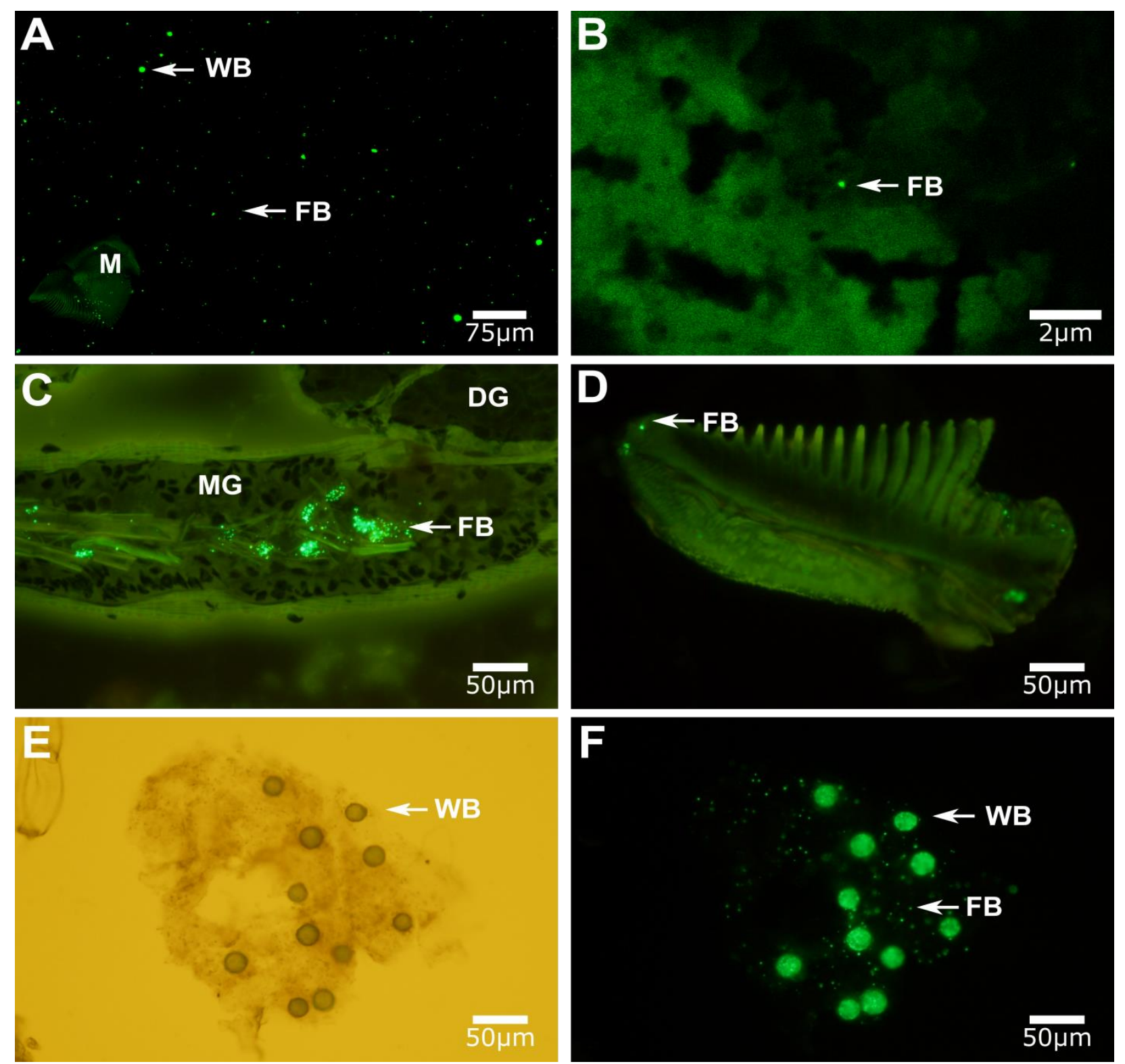

258 Fig. 2. Fate of polyethylene beads and fragments after ingestion by Antarctic krill (Euphausia 259 superba). Krill ( $\mathrm{n}=17)$ were used for histological analysis $\mathrm{A})$ Beads on a filter paper isolated 260 from digested krill with autofluorescent mandible, B) Digestive gland tissue, C) Midgut and 261 digestive gland tissue, D) Mandible with polyethylene fragments embedded in the surface, E) 
and F) Faecal pellet with polyethylene beads under bright field and fluorescence microscopy. WB Whole Bead, FB Fragmented Bead, M Mandible, DG Digestive Gland, MG Midgut
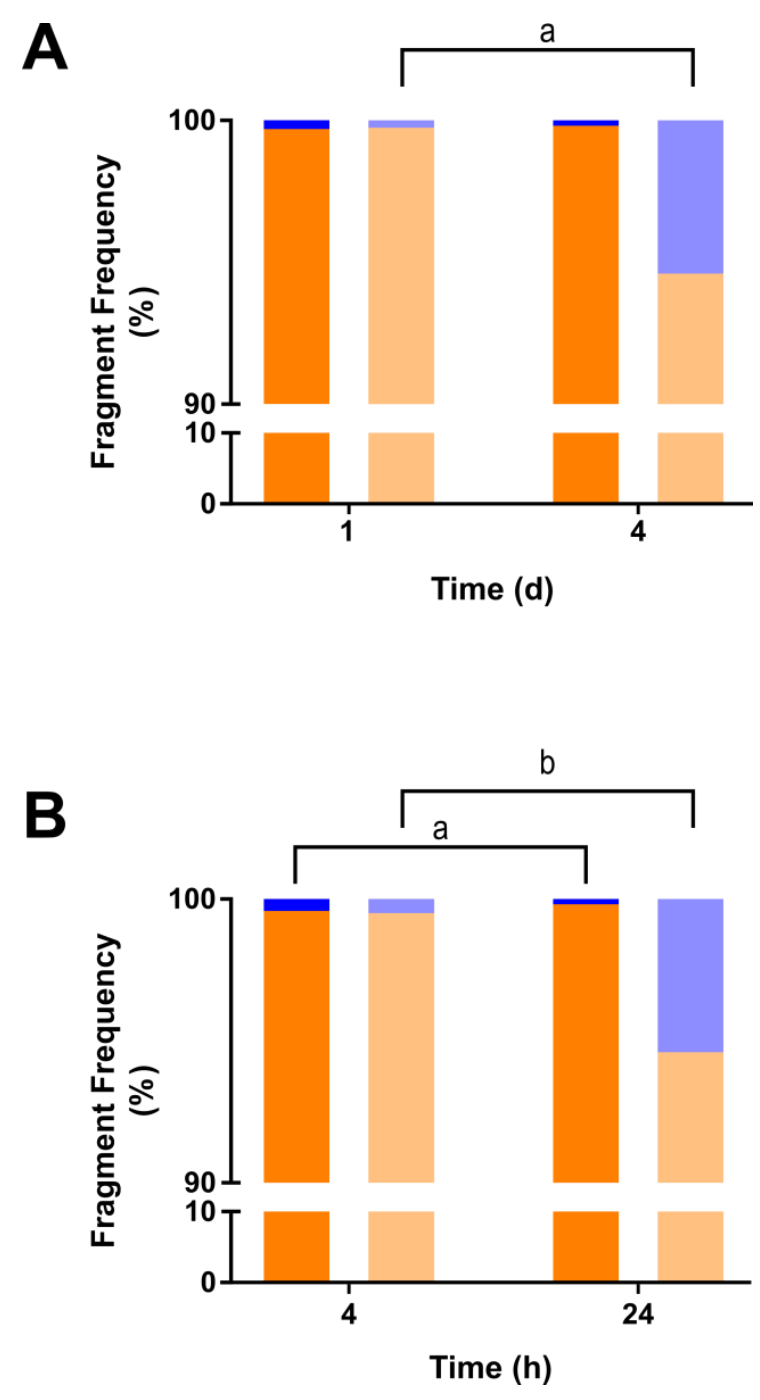

Fig. 3. Frequency of whole (Blue) and fragmented (Orange) particles isolated from faecal 266 pellets of Antarctic krill (Euphausia superba) exposed to Low (20\% - n=3 beakers) (Dark) and High ( $80 \%$ - $n=3$ beakers) (Light) concentrations at: A) 24h on Day $1(n=6)$ and Day 4 $(n=6), B) 4(n=6)$ and $24 h(n=6)$ on Day 4 only. All faecal material per beaker (containing 5 krill) was pooled to form a single sample per time point per dose. Letters denote statistically significance differences in the proportion of whole beads excreted over time $(p<0.05)$. 


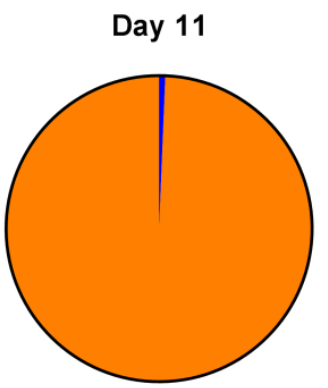

Total $=14740$

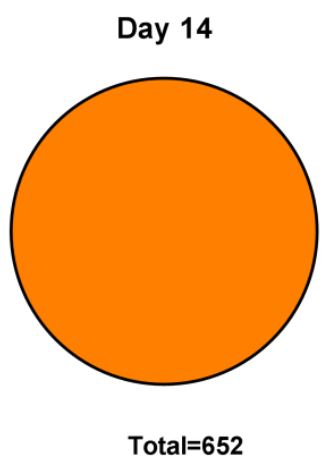

Day 12

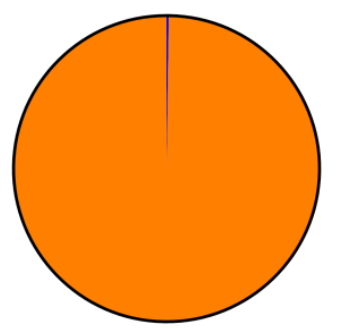

Total $=\mathbf{4 0 4 3}$

Day 15

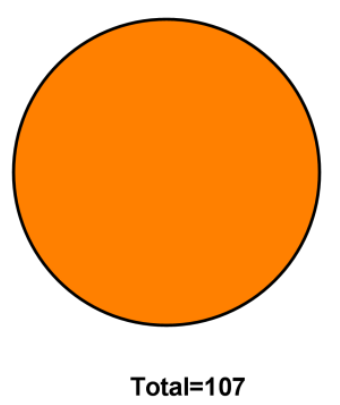

Day 13

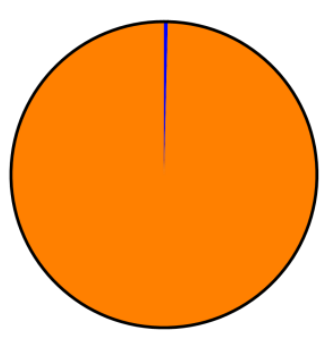

Total $=1983$

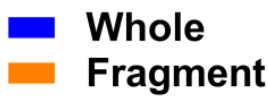

273 Fig. 4. The proportion of PE plastic fragments (Orange) to whole beads (Blue) isolated from Antarctic krill (Euphausia superba) (n=15 krill) faecal material collected over 5 days, after switching from 10 days of low dose microplastic exposure, with daily static renewal, to a clean algae diet. Total refers to the total number of particles measured in each 24 hour period of faecal material. 


\section{Methods}

280 A microplastic feeding stock suspension was made from commercially available (Cospheric LLC CA, 281 USA - UVPMS-BG-1.025) fluorescent green polyethylene microbeads (27-32 $\mu \mathrm{m}$ diameter, 1.030 or $2821.026 \mathrm{~g} \mathrm{~cm}^{-3}$ ). The beads were confirmed to be polyethylene by FTIR spectroscopy using a PerkinElmer 283 FTIR spectrometer (Fig. S4). The bead size range was selected to closely conformed to the size range of the algal food, simultaneously offered to the krill (see below). Density was selected to be close to neutrally buoyant in $0^{\circ} \mathrm{C}$ seawater. The physical properties of the microbeads were characterised using images of beads subsampled from the feeding stock (see Sample Analysis section below).

\section{Exposure Design}

Mixed sex Antarctic krill were collected from the Southern Ocean (66.33 S, 59.34 E) in the Austral summer of 2014/2015. Krill were maintained in the Marine Research Facilities at the Australian Antarctic Division, Tasmania according to previously established methods until use in experiments ${ }^{49}$. Adult krill ( $\mathrm{n}=65$, wet weight: $0.556 \pm 0.117 \mathrm{mg}$, length: $41.1 \pm 3.7 \mathrm{~mm}$; were acclimatised for $24 \mathrm{hrs}$ prior to the start of experiments in $5 \mathrm{~L}$ glass beakers. Krill were randomly selected for use in the experiment from apparently healthy free swimming schooling adults. Krill were collected into buckets by repeatedly dipping a small net into the same region of the tank as the krill schooled anticlockwise. Buckets contained 15 krill; these were randomly distributed amongst beakers, so each beaker contained five adult krill in $4 \mathrm{~L}$ seawater. Block randomisation was applied to distribute krill amongst treatments. The sex of individuals was not determined in the experiment. Seawater temperatures were maintained at $0^{\circ} \mathrm{C}( \pm 0.5)$ and beakers were kept in total darkness throughout the experiment. Exposure seawater was collected from Bruny Island, Tasmania, and filtered to $0.2 \mu \mathrm{m}$. Filtered seawater was pre chilled to $0^{\circ} \mathrm{C}( \pm 0.5)$ before krill were added. The dietary exposure suspension was prepared daily from stock using fluorescent plastic microbeads with concentrated instant non-viable algae Thalassiosira weissflogii (Reed Mariculture Inc, CA, USA). The size range for T. weissflogii cells was 5-20 $\mu \mathrm{m}$ according to the manufacturer. Although this is slightly smaller than the microplastics beads, Antarctic krill can feed efficiently on particles $>2 \mu \mathrm{m}$ up to whole zooplankton $(\sim 3 \mathrm{~mm})$. Dietary exposure suspensions were made up as a portion of the krill's dietary requirements under laboratory conditions, $100 \%$ algae equates to $0.00798 \mathrm{mg} \mathrm{T}$. weissflogii (dry weight) per beaker. Harvested krill were euthanized in liquid nitrogen or formalin. The seawater physiochemical parameters for the two experiments are outlined in Tables S1 and S2.

\section{Particle Size Experiment}

312 Four day feeding and egestion experiments were carried out on 45 Antarctic krill. Nominal daily exposure suspensions were made up to $20 \%$ or $80 \%$ microplastics by weight, which equated to approximately 29 or 116 beads $\mathrm{mL}^{-1}$ (402 or $1606 \mu \mathrm{g} \mathrm{L}^{-1}$ ). Krill were transferred daily to exposure 
suspension and allowed to feed for 4 hours, before being transferred with a stainless steel dip net to a clean beaker for 20 hours. Before transfer, krill were flushed with cold fresh filtered seawater to remove plastics that may adhere to the exoskeleton. Upon transfer to the exposure suspension, krill were observed to be feeding almost immediately. Control krill were fed $100 \%$ algae. Faecal pellets were collected after 24 hours exposure on days 1 and 4 . An extra sample point was added on day 4 to assess fine scale temporal variation after repeated exposure, thus faecal pellets were collected at 4 and 24 hours on day 4 (refer to SI Figure 2). All beakers of krill were harvested for particle size and tissue localisation analysis after 96hrs. Three krill from each beaker were randomly selected for particle size analysis $(\mathrm{n}=18 \mathrm{krill})$. As the beads were fragmented after ingestion, the total bead ingestion rates of could not be calculated from stomach content or egested material.

\section{Tissue Localisation Experiment}

To investigate tissue localisation of ingested plastic, two krill from each beaker were randomly selected, fixed in formalin, and used for histological cryo-section $(20 \mu \mathrm{m})$ analysis. Slides were stained with H\&E or remained unstained. Slides were examined using an Olympus BX60 fluorescence microscope or Zeiss-780 Laser Scanning Confocal microscope with a fluorescent filter of $488 \mathrm{~nm}$ excitation and 526 $\mathrm{nm}$ emission.

In addition, to investigate if krill could fragment plastics $<1 \mu \mathrm{m}$ and the possibility of fragments entering the digestive gland, five krill were exposed to $100 \%$ plastic diet (approx. $2063 \mu \mathrm{g} \mathrm{L}^{-1}$ or 149 beads $\mathrm{mL}^{-}$ $\left.{ }^{1}\right)$ for $24 \mathrm{hrs}$, with no water changes. These 5 krill were all used for tissue localisation analysis.

\section{Egestion Experiment}

335 To examine particle sizes egested over an extended period, 15 krill divided into 3 beakers were exposed 336 for 10 days to a $20 \%$ diet (approx. $401 \mu \mathrm{g} \mathrm{L}^{-1}$ ) of plastic following the same basic design as the Particle 337 Size Experiment. After 10 days, the diet was switch to $100 \%$ algae for five days. Faecal pellets were 338 collected at 4 and 24 hours every day of the five day egestion period. Faecal material was pooled per 339 beaker per 24 hours resulting in 15 samples.

\section{Sample Analysis}

341 Body burden analysis was carried out using an enzyme digestion followed by visual identification of ingested microplastics under a fluorescent microscope. Krill were flushed with milli-q water, blotted dry, weighed (to 3 d.p), and heated to $65^{\circ} \mathrm{C}$ in a water bath, after which the exoskeleton was removed.

344 Krill were then homogenised using a glass rod, and digested using proteinase $\mathrm{K}$ adapted from ${ }^{50}$, which was previously shown to have negligible effects on PS bead integrity. Digestion efficacy was not optimal as hard chitinous structures often remained after digestion. Digested krill were filtered under vacuum onto Millipore gridded $0.45 \mu \mathrm{m}$ filters and air dried overnight. Filters were fixed between glass coverslips and analysed for microplastics using a Zeiss-780 Laser Scanning Confocal microscope with a fluorescent filter with a Plan-Apochromat 10X/0.45 M27 lens, with a numerical aperture of 0.45 . Microplastic fragments were imaged in five randomly selected squares $(6.97 \times 6.98 \mathrm{~mm}$; total area of 
$2.4 \mathrm{~cm}^{2}$ ) on the filter paper, which accounted for $25 \%$ of the total filtered area. Images were verified by eye, and compared to controls to examine for undigested chitinous material with autofluorescence. Of the 165 images taken, 2 images were excluded on the basis of chitinous material with autofluorescence (See Fig 2. panel A for example of excluded image). These were too large to be mistaken as a microplastic beads and were clearly distinguishable as mandibles. The diameter (major axis when particles were fitted to an ellipse) of each particle within each image was measured using imaging software (FIJI GPL v2) ${ }^{51}$. A minimum threshold was applied to the fluorescence intensity of each image to ensure only beads were counted by the imaging software. Thresholds were set to a minimum of 65 and maximum of 255 which allowed background material, including undigested exoskeleton (except for mandibles), algal cells and the filter paper, to be excluded without interference to the analysis. Size exclusions were applied to particles which had a diameter $>50 \mu \mathrm{m}$, on the basis these were 2 or more beads too close together for the imaging software to distinguish individual beads and accurately measure size.

\section{Bead fragmentation}

To test that the sample analysis procedures were not responsible for fragmenting the beads, procedural blanks were carried out in a pilot study and throughout the experiment. Procedural blanks consisted of krill and beads or just beads. Krill blanks consisted of 7 krill taken from a pilot study. The krill were digested as per the method described in the Sample Analysis section, except the krill were not homogenised. After digestion, exoskeleton remained intact but the tissue was completely digested, krill were vortexed and the stomach was opened to liberate any remaining beads. The sample was then filtered and imaged as per the method described in the Sample Analysis section. Bead blanks consisted of beads in the absence of krill and were not homogenised. Beads were added to buffer and enzyme, then digested, filtered and imaged as per methods outlined in the Sample Analysis section. Bead blanks were examined after enzyme digestion with FTIR spectroscopy (Fig. S5), but ingested beads and fragments were unable to be detected on the cellulose filters with FTIR due to the low concentration and/or small size of the particle.

\section{Statistical Analysis}

Two-sample Kolmogorov-Smirnov Tests (two tailed, $\alpha \leq 0.05$ ) were used to compare the particle size distribution from the stock microbeads to the size distribution of plastics isolated from the digested krill, and from the particles isolated from the faecal pellets. The proportion of whole beads compared to fragments in digested krill and in faecal pellets was compared between doses using Chi squared analysis (two tailed, $\alpha \leq 0.05$ ). For all proportion tests (Chi squared and linear regression), beads with a diameter $\geq 25 \mu \mathrm{m}$ were classified as whole beads, beads $<25 \mu \mathrm{m}$ were considered fragments. This cut off was selected by eye using the standard distribution of the stock beads. Kolmogorov-Smirnov Tests (two tailed, $\alpha \leq 0.05$ ) were used to test for normality. The data was $\log _{10}$ transformed and comparison between fragment size, 
sample time and plastic dose in the faecal pellets was determined with a two-way ANOVA (two tailed, $\alpha \leq 0.05$ ). Multiple linear regression was used to examine relationships between the length and weight of the krill and their ability to fragment plastics. Means are expressed as mean \pm standard deviation (S.D) unless otherwise stated.

\section{Data Availability}

Acknowledgments: A.D was supported by supported by PHD scholarship from ARC Discovery DP140100018. We thank S. Wild and P. Eisenmann for assistance with laboratory work, and staff at the Australian Antarctic Division for maintenance of krill in the Marine Research Facilities. We thank M. Arthur and C. Wild for providing statistical advice. N. Waterhouse for assisting design of imaging methods, T. Nguyen for imaging samples and staff at the QIMR Berghofer Medical Research Institute for sectioning the krill.

Author Contributions: A.D and S.BN conceived of the idea of this study and provided financial means. A.D and C.K preformed laboratory experiments. R.K and A.D interpreted histological data. W.H and A.D designed image analysis methods. A.D analysed the data. A.D prepared the manuscript, all authors contributed substantially to revisions.

\section{References}

1 Wesch, C., Bredimus, K., Paulus, M. \& Klein, R. Towards the suitable monitoring of ingestion of microplastics by marine biota: A review. Environ Pollut, doi:10.1016/j.envpol.2016.08.076 (2016).

2 Bergmann, M., Gutow, L. \& Klages, M. Marine anthropogenic litter. (Springer, 2015).

3 Phuong, N. N. et al. Is there any consistency between the microplastics found in the field and those used in laboratory experiments? Environ Pollut 211, 111-123, doi:10.1016/j.envpol.2015.12.035 (2016).

4 Setälä, O., Norkko, J. \& Lehtiniemi, M. Feeding type affects microplastic ingestion in a coastal invertebrate community. Mar Pollut Bull 102, 95-101, doi:10.1016/j.marpolbul.2015.11.053 (2016).

5 Andrady, A. L. Microplastics in the marine environment. Mar Pollut Bull 62, 1596-1605, doi:10.1016/j.marpolbul.2011.05.030 (2011).

6 Welden, N. A. C. \& Cowie, P. R. Long-term microplastic retention causes reduced body condition in the langoustine, Nephrops norvegicus. Environ Pollut 218, 895-900, doi:10.1016/j.envpol.2016.08.020 (2016).

7 Cole, M. et al. Microplastic Ingestion by Zooplankton. Environ Sci Technol 47, 6646-6655, doi:10.1021/es400663f (2013).

8 Hämer, J., Gutow, L., Köhler, A. \& Saborowski, R. Fate of Microplastics in the Marine Isopod Idotea emarginata. Environ Sci Technol 48, 13451-13458, doi:10.1021/es501385y (2014). 
Kaposi, K. L., Mos, B., Kelaher, B. P. \& Dworjanyn, S. A. Ingestion of microplastic has limited impact on a marine larva. Environ Sci Technol 48, 1638-1645, doi:10.1021/es404295e (2014).

Setälä, O., Fleming-Lehtinen, V. \& Lehtiniemi, M. Ingestion and transfer of microplastics in the planktonic food web. Environ Pollut 185, 77-83, doi:10.1016/j.envpol.2013.10.013 (2014).

Cole, M. \& Galloway, T. S. Ingestion of Nanoplastics and Microplastics by Pacific Oyster Larvae. Environ Sci Technol 49, 14625-14632, doi:10.1021/acs.est.5b04099 (2015).

Bergami, E. et al. Nano-sized polystyrene affects feeding, behavior and physiology of brine shrimp Artemia franciscana larvae. Ecotoxicol Environ Saf 123, 18-25, doi:10.1016/j.ecoenv.2015.09.021 (2016).

Besseling, E., Wang, B., Lürling, M. \& Koelmans, A. A. Nanoplastic Affects Growth of S. obliquus and Reproduction of D. magna. Environ Sci Technol 48, 12336-12343, doi:10.1021/es503001d (2014).

Lee, K.-W., Shim, W. J., Kwon, O. Y. \& Kang, J.-H. Size-Dependent Effects of Micro Polystyrene Particles in the Marine Copepod Tigriopus japonicus. Environ Sci Technol 47, 11278-11283, doi:10.1021/es401932b (2013).

Rehse, S., Kloas, W. \& Zarfl, C. Short-term exposure with high concentrations of pristine microplastic particles leads to immobilisation of Daphnia magna. Chemosphere 153, 91-99, doi:10.1016/j.chemosphere.2016.02.133 (2016).

Schmidt, K., Atkinson, A., Petzke, K.-J., Voss, M. \& Pond, D. W. Protozoans as a food source for Antarctic krill, Euphausia superba : Complementary insights from stomach content, fatty acids, and stable isotopes. Limnol Oceanogr 51, 2409-2427 (2006).

Everson, I. Krill: Biology, Ecology and Fisheries. (Blackwell Science Ltd, 2000). Siegel, V. Biology and Ecology of Antarctic Krill. (Springer International Publishing, 2016).

Smetacek, V., Assmy, P. \& Henjes, J. The role of grazing in structuring Southern Ocean pelagic ecosystems and biogeochemical doi:10.1017/S0954102004002317 (2004).

Boyd, C. M., Heyraud, M. \& Boyd, C. N. Feeding of the Antarctic krill Euphausia superba. J Crustacean Biol 4, 123-141, doi:10.2307/27920092 (1984).

Hamner, W. M. Biomechanics of Filter Feeding in the Antarctic Krill Euphausia superba: Review of past Work and New Observations. J Crustacean Biol 8, 149-163, doi:10.2307/1548308 (1988).

McClatchie, S. \& Boyd, C. M. Morphological Study of Sieve Efficiencies and Mandibular Surfaces in the Antarctic Krill, Euphausia superba. Can J Fish Aquat Sci 40, 955-967, doi:10.1139/f83-122 (1983).

Suh, H.-L. \& Nemoto, T. Morphology of the gastric mill in ten species of euphausiids. Mar. Biol. 97, 79-85, doi:10.1007/bf00391247 (1988).

Ikeda, T., Nash, G. V. \& Thomas, P. G. An observation of discarded stomach with exoskeleton moult from Antarctic krill Euphausia superba Dana. Polar Biol. 3, 241-244, doi:10.1007/bf00292631 (1984).

Ullrich, B., Storch, V. \& Marschall, H. P. Microscopic anatomy, functional morphology, and ultrastructure of the stomach of Euphausia superba Dana (Crustacea, Euphausiacea). Polar Biol. 11, 203-211, doi:10.1007/bf00240209 (1991).

6 Dall, W. \& Moriarty, D. J. W. in Internal Anatomy and Physiological Regulation Vol. 5 (ed Linda Mantel) Ch. 4, 215-261 (Academic Press, 1983). 
Spicer, J. I. \& Saborowski, R. Physiology and Metabolism of Northern Krill (Meganyctiphanes norvegica Sars). Adv. Mar. Biol. 57, 91-126, doi:10.1016/B978-0-12-381308-4.00004-2 (2010).

Saborowski, R. \& Buchholz, F. A laboratory study on digestive processes in the Antarctic krill, Euphausia superba, with special regard to chitinolytic enzymes. Polar Biol. 21, 295-304, doi:10.1007/s003000050365 (1999).

Bliss, D. E. The Biology of Crustacea: Internal anatomy and physiological regulation. (Academic Press, 1982).

Singh, A. K. Engineered Nanoparticles: Structure, Properties and Mechanisms of Toxicity. (Elsevier Science, 2015).

Ward, J. E. \& Kach, D. J. Marine aggregates facilitate ingestion of nanoparticles by suspensionfeeding bivalves. Mar Environ Res 68, 137-142, doi:10.1016/j.marenvres.2009.05.002 (2009). Van Cauwenberghe, L. \& Janssen, C. R. Microplastics in bivalves cultured for human consumption. Environ Pollut 193, 65-70, doi:10.1016/j.envpol.2014.06.010 (2014).

Rosenkranz, P., Chaudhry, Q., Stone, V. \& Fernandes, T. F. A comparison of nanoparticle and fine particle uptake by Daphnia magna. Environ Toxicol Chem 28, 2142-2149, doi:10.1897/08559.1 (2009).

Desforges, J.-P. W., Galbraith, M. \& Ross, P. S. Ingestion of Microplastics by Zooplankton in the Northeast Pacific Ocean. Arch Environ Contam Toxicol 69, 320-330, doi:10.1007/s00244015-0172-5 (2015).

Reisser, J., Proietti, M., Shaw, J. \& Pattiaratchi, C. Ingestion of plastics at sea: does debris size really matter? Frontiers in Marine Science 1, doi:10.3389/fmars.2014.00070 (2014).

Odian, G. Principles of Polymerization. Vol. 4 (John Wiley \& Sons, Inc., 2004).

Rocha-Santos, T. A. P. \& Duarte, A. C. Characterization and Analysis of Microplastics. Vol. 75 (Elsevier Science, 2017).

39 ter Halle, A. et al. To what extent are microplastics from the open ocean weathered? Environ

40 Watts, A. J. R., Urbina, M. A., Corr, S., Lewis, C. \& Galloway, T. S. Ingestion of Plastic Microfibers by the Crab Carcinus maenas and Its Effect on Food Consumption and Energy Balance. Environ Sci Technol 49, 14597-14604, doi:10.1021/acs.est.5b04026 (2015).

Cózar, A. et al. Plastic debris in the open ocean. Proceedings of the National Academy of Sciences 111, 10239-10244, doi:10.1073/pnas.1314705111 (2014).

Eriksen, M. et al. Plastic Pollution in the World's Oceans: More than 5 Trillion Plastic Pieces Weighing over 250,000 Tons Afloat at Sea. PLoS One 9, e111913, doi:10.1371/journal.pone.0111913 (2014).

Cincinelli, A. et al. Microplastic in the surface waters of the Ross Sea (Antarctica): Occurrence, distribution and characterization by FTIR. Chemosphere 175, 391-400, doi:10.1016/j.chemosphere.2017.02.024 (2017).

Isobe, A., Uchiyama-Matsumoto, K., Uchida, K. \& Tokai, T. Microplastics in the Southern Ocean. Mar Pollut Bull 114, 623-626, doi:10.1016/j.marpolbul.2016.09.037 (2017). 
50845 Browne, M. A., Dissanayake, A., Galloway, T. S., Lowe, D. M. \& Thompson, R. C. Ingested 509 Microscopic Plastic Translocates to the Circulatory System of the Mussel, Mytilus edulis (L.). $510 \quad$ Environ Sci Technol 42, 5026-5031, doi:10.1021/es800249a (2008).

$51146 \quad$ Farrell, P. \& Nelson, K. Trophic level transfer of microplastic: Mytilus edulis (L.) to Carcinus 512 maenas (L.). Environ Pollut 177, 1-3, doi:10.1016/j.envpol.2013.01.046 (2013).

$51347 \quad$ Avio, C. G. et al. Pollutants bioavailability and toxicological risk from microplastics to marine $514 \quad$ mussels. Environ Pollut 198, 211-222, doi:10.1016/j.envpol.2014.12.021 (2015).

51548 ter Halle, A. et al. Understanding the Fragmentation Pattern of Marine Plastic Debris. Environ 516 Sci Technol 50, 5668-5675, doi:10.1021/acs.est.6b00594 (2016).

$51749 \quad$ Kawaguchi, S. et al. An experimental aquarium for observing the schooling behaviour of $518 \quad$ Antarctic krill (Euphausia superba). Deep-Sea Res Part II Top Stud Oceanogr 57, 683-692, 519 doi:10.1016/j.dsr2.2009.10.017 (2010).

52050 Cole, M. et al. Isolation of microplastics in biota-rich seawater samples and marine organisms. $521 \quad$ Sci. Rep. 4, doi:10.1038/srep04528 (2014).

$52251 \quad$ Schindelin, J. et al. Fiji: an open-source platform for biological-image analysis. Nat. Methods 9, 676-682, doi:10.1038/nmeth.2019 (2012). 